\title{
Acute Pharmacological DVT Prophylaxis after Spinal Cord Injury
}

\author{
Sean Christie, Ginette Thibault-Halman, ${ }^{2}$ and Steven Casha ${ }^{1}$
}

\begin{abstract}
A systematic review of the literature was performed to address pertinent clinical questions regarding deep vein thrombosis (DVT) prophylaxis in the setting of acute spinal cord injury (SCI). Deep vein thromboses are a common occurrence following SCI. Administration of low-molecular-weight heparin (LMWH) within $72 \mathrm{~h}$ of injury is recommended to minimize the occurrence of DVT. Furthermore, when surgical intervention is required, LMWH should be held the morning of surgery, and resumed within $24 \mathrm{~h}$ post-operatively.
\end{abstract}

Key words: deep vein thrombosis; spinal cord injury

\section{Introduction}

A COMMON COMPLICATION following spinal cord injury (SCI) IS DEEP VEIN THROMBOSIS (DVT). The incidence of DVT that has been reported in the literature varies widely depending on the screening tool, with rates ranging from 9-100\% (Teasell et al., 2008). Venous thromboses are a significant cause of morbidity and mortality, and are responsible for $9.7 \%$ of all deaths in the first year following SCI (DeVivo et al., 1999). In fact, within the first month postinjury, SCI patients have a 500-fold increased risk of PErelated death relative to age- and gender-matched noninjured controls (DeVivo et al., 1989). This vulnerability to DVT may be due in part to the presence of Virchow's triad of stasis, hypercoagulability, and vessel intimal injury (Furlan and Fehlings, 2008). Additional pathophysiological mechanisms have also been implicated, including impaired circadian variations of hemostatic and fibrinolytic parameters, as well as changes in platelet function and fibrinolytic activity (Iversen et al., 2002; Winther et al., 1992).

Strategies used for the prevention of DVT have included oral anticoagulants, heparin (both unfractionated and lowmolecular-weight heparin), antithromoboembolic stockings, external compression devices, and vena cava filters. Among the pharmacological approaches, a previous systematic review has suggested that low-molecular-weight heparin (LMWH) is more effective than standard or unfractionated heparin in preventing DVT in the SCI population (Teasell et al., 2008); however, the optimal timing of initiation of LMWH administration has not yet been established. The following clinically relevant question regarding DVT prophylaxis following acute SCI was used to guide this systematic review: What is the ideal time for initiation of DVT prophylaxis with LMWH: after SCI or after surgery?

\section{Methods}

\section{Search strategy}

A primary literature search was performed using PubMed. The search was conducted for the following terms: "thromboembolism," "VT," "DVT," "venous thrombosis," and "deep vein thrombosis." These key words were then paired with "SCI," "spinal cord injuries," and "spinal cord injury," as well as the MeSH term "Spinal Cord Injuries." The search was limited to English language articles and research involving human subjects. Given the advantage of LMWH over unfractionated heparin (Teasell et al., 2008), this review focused on studies utilizing only LMWH.

Articles included were those that addressed the timing of LMWH thromboprophylaxis initiation, or those that described LMWH administration in the perioperative period (i.e., whether the dose was held preoperatively, and the timeline of resumption of LMWH). Articles excluded were those that did not address either efficacy or complication rates; those that focused on unfractionated heparin rather than LMWH; any articles not related significantly to SCI (i.e., mixed trauma patients in whom the majority of patients did not have an SCI); any articles specific for chronic SCI; any single-case reports; any commentaries, opinions, or reviews; and mainly pediatric reports. Key reviewed articles were examined for more papers.

\section{Article review process}

Relevant articles from the search were rated by two independent reviewers according to Downs and Black scoring scheme (Downs and Black, 1998). Any variance in the scoring between the two reviewers was addressed by a third reviewer. Data were presented to the Spinal Cord Injury

\footnotetext{
${ }^{1}$ Division of Neurosurgery, Dalhousie University, Halifax, Nova Scotia, Canada.

${ }^{2}$ Division of Neurosurgery, Capital District Health Authority, Halifax Infirmary, Halifax, Nova Scotia, Canada.
} 
Solutions Network (Acute Practice Network), and subjected to a modified Delphi review process to establish an expert consensus to address the guiding question.

\section{Results}

In all 312 articles were returned by the search. Abstracts were screened, and five met inclusion/exclusion criteria. The relevant articles were then subjected to scoring according to the Downs and Black criteria (Table 1)

\section{What is the ideal time for initiation of DVT prophylaxis with LMWH: after SCl or after surgery?}

Only one of the studies reviewed was specifically designed to determine the effects of the timing of prophylaxis post-SCI. However, four other studies that met the inclusion/exclusion criteria did specify when DVT prophylaxis was administered relative to the time of injury, and addressed efficacy and/or complications.

Aito and colleagues compared an early group (DVT prophylaxis within $72 \mathrm{~h}$, including $\mathrm{LMWH}$ ) to a late group (prophylaxis started after $72 \mathrm{~h}$ ). DVT incidence was based upon color Doppler ultrasonography, which was performed on admission, at 45-60 days, and whenever clinically indicated. The incidence of DVT in the early group was $2 \%$, whereas the late group had an incidence of $26 \%$ (Aito et al., 2002).

Green and associates conducted a study designed to compare LMWH to unfractionated heparin. They initiated LMWH DVT prophylaxis within $72 \mathrm{~h}$ of injury. Prophylaxis was held on the day of surgery and resumed the next morning. They found a 3.3\% rate of pulmonary embolism (PE) and a $10 \%$ rate of DVT, as measured by ultrasound at 8 weeks (upon completion of LMWH), and every week thereafter for 4 weeks, as well as daily clinical exams for signs and symptoms of thromboembolism. Only one complication was reported, consisting of bleeding in a patient with pre-existing hematuria secondary to a gunshot wound (Green et al., 1994).

An earlier study by Green and associates was also designed to compare LMWH to unfractionated heparin (Green et al., 1990). Patients were enrolled within $72 \mathrm{~h}$ of injury; LMWH was not initiated until at least $24 \mathrm{~h}$ after injury. Thromboprophylaxis was held the morning of surgery, and resumed the day following surgery. Daily clinical assessments as well as serial venous flow studies were used to detect thromboembolism. Among the 20 patients in this study receiving $\mathrm{LWMH}$, none had PE or DVT, nor did any have significant bleeding. (Conversely, the cumulative event rate for bleeding or DVT was $34.7 \%$ among the standard heparin group.)

A randomized controlled trial (comparing unfractioned heparin and intermittant compression stockings vs. enoxaparin) followed the same timeline of LMWH administration, instituting prophylaxis within $72 \mathrm{~h}$, holding the drug on the day of surgery, and resuming treatment the first postoperative morning. Patients were assessed for DVT using Doppler ultrasound at 14 days post-injury, and for PE via ventilation-perfusion scan, spiral CT, or pulmonary angiography. The rates of PE and DVT among those on LMWH were $5.2 \%$ and $60.3 \%$, respectively; however, the rate of symptomatic DVT was just 1.7\%. Minor bleeding occurred in $14.8 \%$ of patients receiving enoxaparin, with a rate of major bleeding of $2.6 \%$ (Spinal Cord Injury Thromboprophylaxis Investigators, 2003).

Harris and co-workers completed a retrospective chart review of 105 patients who received LMWH post-SCI beginning at the time of admission. The drug was held the morning of surgery, and resumed $24 \mathrm{~h}$ later (Harris et al., 1996). There was no clinical evidence of DVT in any of the patients reviewed; among the 60 patients who underwent ultrasonography, there was no evidence of DVT, although $11 / 105$ patients had some bleeding as evidenced by a decline in hemoglobin of $2 \mathrm{~g} / \mathrm{dL}$ or greater. Enoxaparin was found to be contributory in three of these cases. The drug continued to be administered in all but two of the patients (one with bleeding cause unknown, the other with a hematoma secondary to a gunshot wound), yet there was no evidence that continued administration led to exacerbation of the bleeding. Although there were five deaths, none were attributable to DVT prophylaxis.

\section{Discussion}

In the absence of thromboprophylaxis, SCI patients have a higher incidence of DVT than any other hospitalized group (Geerts, 2008). This patient population also presents a diagnostic challenge, since thromboembolism can often be clinically silent or difficult to distinguish from other common SCI complications (Chen, 2003). Nonpharmacological treatment modalities such as early mobilization can be hampered by associated injuries, respiratory insufficiency, or spinal instability (Chen, 2003).

Regarding pharmacological management, it has been previously established that LMWH is preferable to unfractionated heparin in the SCI population due to its longer half-life, lower risk of bleeding complications, and more predictable dose effect relative to unfractionated heparin (Teasell et al., 2008). Thus in this review we focused our attention on the optimal timing of prophylaxis administration. Although only one study has examined this directly (Aito et al., 2002), the difference between early administration and administration later than $72 \mathrm{~h}$ was large ( $2 \%$ and $26 \%$ incidence of DVT, respectively). Complication rates associated with LMWH were relatively low $(1.6-10 \%)$, including when thromboprophylaxis was resumed within $24 \mathrm{~h}$ post-operatively (Green et al., 1990, 1994; Harris et al., 1996; Spinal Cord Injury Thromboprophylaxis Investigators, 2003). This parallels recommendations for the major trauma population, in whom thromboprophylaxis should be initiated as soon as it considered safe to do so (in the absence of contraindications) (Geerts, 2008).

\section{Systematic review recommended answers to the clinical question: What is the ideal time for initiation of DVT prophylaxis with LMWH: After $\mathrm{SCl}$ or after surgery?}

- DVT prophylaxis should be instituted within $72 \mathrm{~h}$ postinjury. (Single study but large difference; therefore suggest strong recommendation with weak data.)

- LMWH should be held on the morning of surgery and resumed within $24 \mathrm{~h}$ following surgery. (Balance of risks and benefits; strong recommendation, weak data.) 


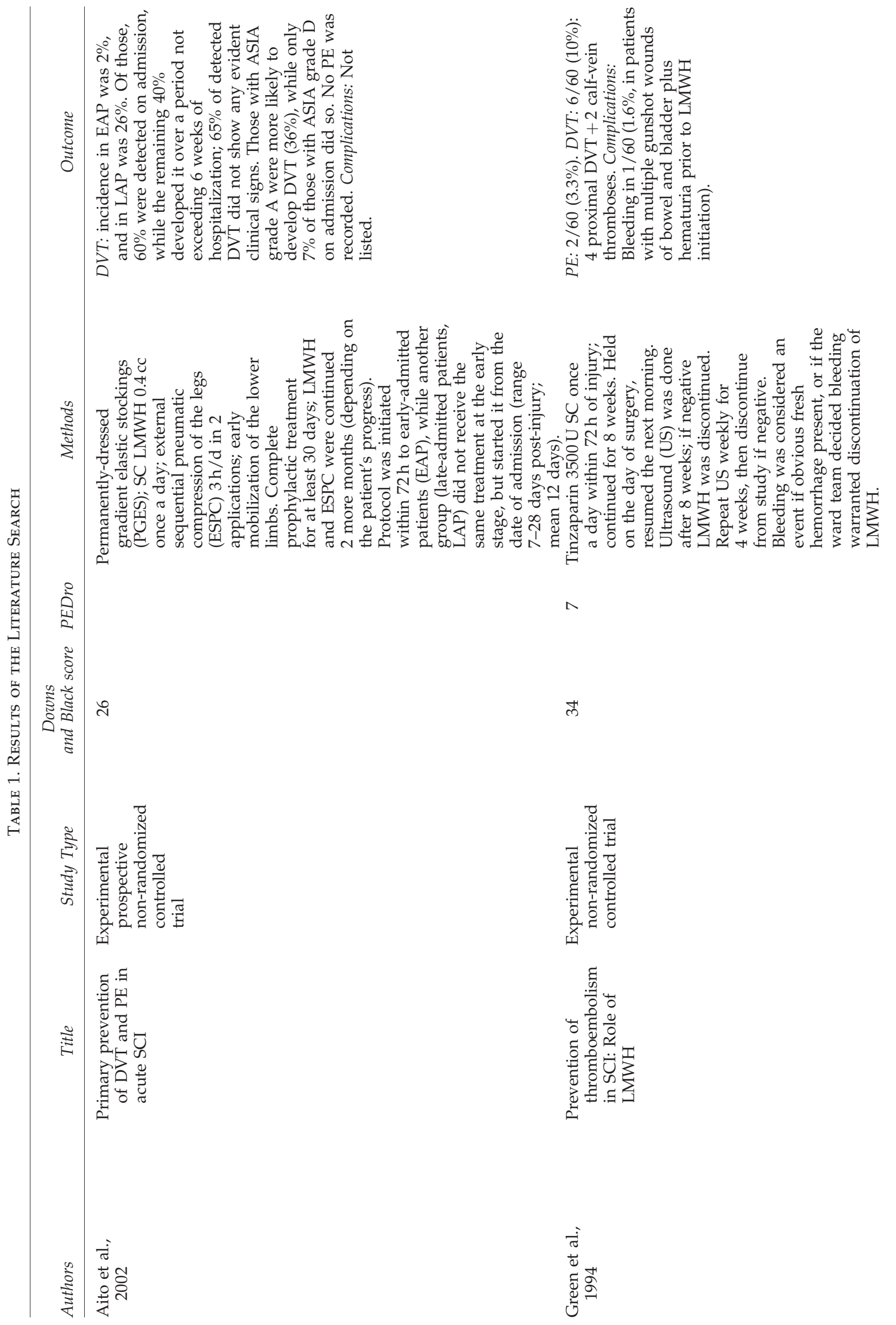




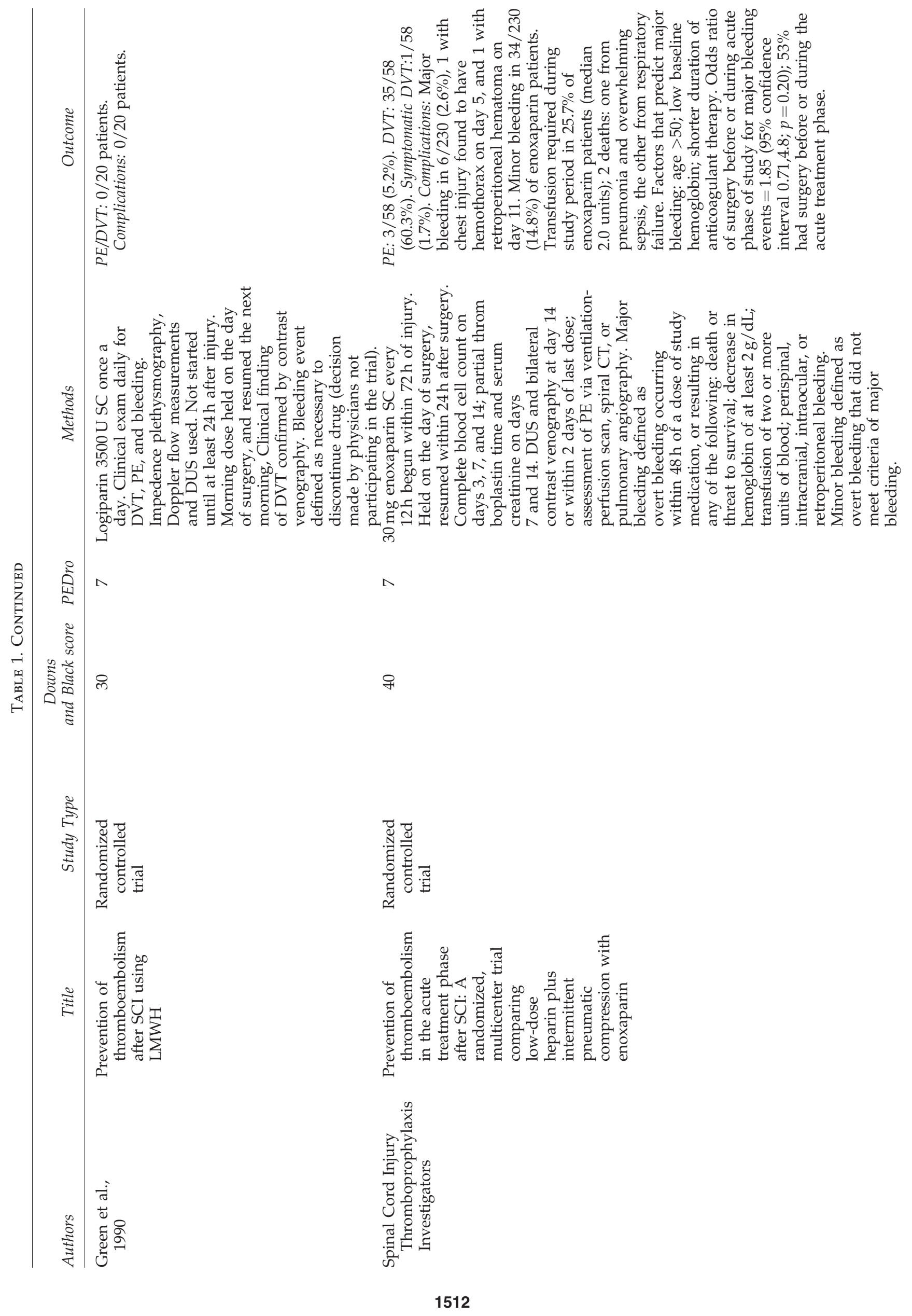




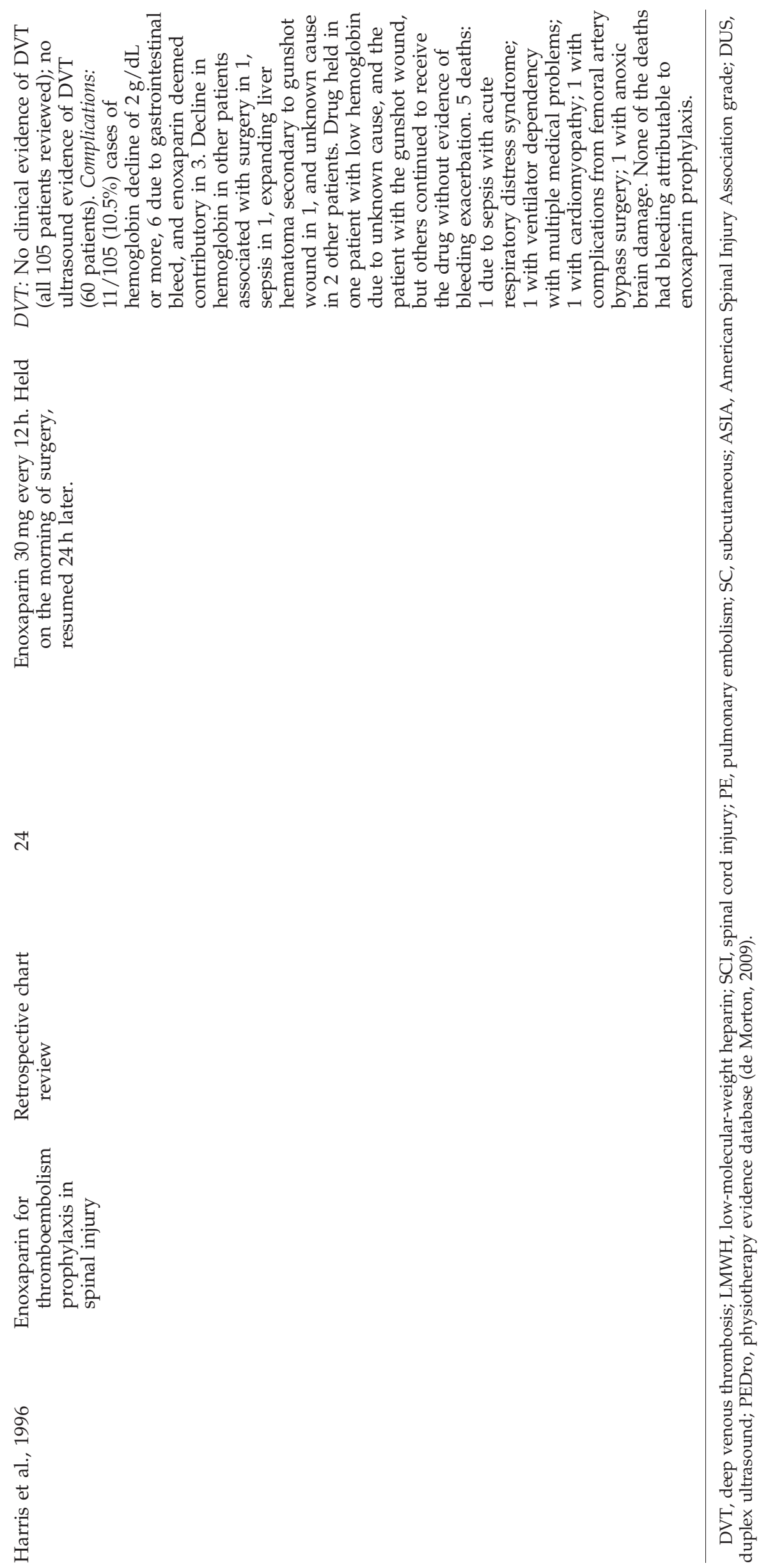




\section{Author Disclosure Statement}

No competing financial interests exist.

\section{References}

Aito, S., Pieri, A., D'Andrea, M., Marcelli, F., and Cominelli, E. (2002). Primary prevention of deep venous thrombosis and pulmonary embolism in acute spinal cord injured patients. Spinal Cord 40, 300-303.

Chen, D. (2003). Treatment and prevention of thromboembolism after spinal cord injury. Top Spinal Cord Inj Rehabil 9, 14.

de Morton, N.A. (2009). The PEDro scale is a valid measure of the methodological quality of clinical trials: A demographic study Aust J Physiother. 55, 129-133.

DeVivo, M.J., Kartus, P.L., Stover, S.L., Rutt, R.D., and Fine, P.R. (1989). Cause of death for patients with spinal cord injuries. Arch. Intern. Med. 149, 1761-1766.

DeVivo, M.J., Krause, J.S., and Lammertse, D.P. (1999). Recent trends in mortality and causes of death among persons with spinal cord injury. Arch. Phys. Med. Rehabil. 80, 1411-1419.

Downs, S.H., and Black, N. (1998). The feasibility of creating a checklist for the assessment of the methodological quality both of randomised and non-randomised studies of health care interventions. J. Epidemiol. Community Health 52, 377-384.

Furlan, J.C., and Fehlings, M.G. (2008). Cardiovascular complications after acute spinal cord injury: pathophysiology, diagnosis, and management. Neurosurg. Focus 25, E13.

Geerts, W.H., Bergqvist, D., Pineo, G.F., Hett, J.A., Samama, C.M., Lassen, M.R., Colwell, C.W., and American College of Chest Physicians. (2008). Prevention of venous thromboembolism: American College of Chest Physicians Evidence-Based Clinical Practice Guidelines (8th Edition) Chest. 133, 381S-453S.

Green, D., Chen, D., Chmiel, J.S., Olsen, N.K., Berkowitz, M., Novick, A., Alleva, J., Steinberg, D., Nussbaum, S., and Tolotta, M. (1994). Prevention of thromboembolism in spinal cord injury: role of low molecular weight heparin. Arch. Phys. Med. Rehabil. 75, 290-292.
Green, D., Lee, M.Y., Lim, A.C., Chmiel, J.S., Vetter, M., Pang, T., Chen, D., Fenton, L., Yarkony, G.M., and Meyer, P.R. Jr. (1990). Prevention of thromboembolism after spinal cord injury using low-molecular-weight heparin. Ann. Intern. Med. $113,571-574$.

Harris, S., Chen, D., and Green, D. (1996). Enoxaparin for thromboembolism prophylaxis in spinal injury: preliminary report on experience with 105 patients. Am. J. Phys. Med. Rehabil. 75, 326-327.

Iversen, P.O., Groot, P.D., Hjeltnes, N., Andersen, T.O., Mowinckel, M.C., and Sandset, P.M. (2002). Impaired circadian variations of haemostatic and fibrinolytic parameters in tetraplegia. Br. J. Haematol. 119, 1011-1016.

Spinal Cord Injury Thromboprophylaxis Investigators. (2003). Prevention of venous thromboembolism in the acute treatment phase after spinal cord injury: a randomized, multicenter trial comparing low-dose heparin plus intermittent pneumatic compression with enoxaparin. J. Trauma 54, 1116-1124; discussion 1125-1126.

Teasell, R.W., Hsieh, J.T.C., Aubut, J., Eng, J.J., Krassioukov, A., and $\mathrm{Tu}, \mathrm{L}$. (2008). Venous thromboembolism following spinal cord injury, in: Spinal Cord Injury Rehabilitation Evidence, 20th ed. J.J. Eng, R.W. Teasell, D.L. Wolfe, et al. (eds), pps. 15. $1-15.27$.

Winther, K., Gleerup, G., Snorrason, K., and Biering-Sorensen, F. (1992). Platelet function and fibrinolytic activity in cervical spinal cord injured patients. Thromb. Res. 65, 469-474.

Address correspondence to: Sean Christie, M.D., FRCSC

Division of Neurosurgery Dalhousie University 3814-1796 Summer Street Halifax, Nova Scotia, Canada B3H 3 A7

E-mail: sean.christie@dal.ca 